\title{
Seroprevalence of Leptospirosis in Suspected High Risk Sudanese Patients; A Pilot Exploratory Study
}

\section{Wisal Mustafa Ibrahim Mohammed}

University of Medical Sciences and Technology

Nada Abdelghani Abdelrahim ( $\nabla$ nada.ghani@hotmail.com )

Department of Medical Microbiology, Faculty of Medical Laboratory Sciences, Nile University https://orcid.org/0000-0001-8736-1148

\section{Short Report}

Keywords: Prevalence, IgM Antibodies, Leptospiral Infection, Renal Dialysis, Fever, Sudan

Posted Date: January 11th, 2022

DOI: https://doi.org/10.21203/rs.3.rs-1246367/v1

License: (1) This work is licensed under a Creative Commons Attribution 4.0 International License. Read Full License 


\section{Abstract}

\section{Background}

The extent of leptospirosis is unknown in Sudan and it might be mistaken for other more common febrile infectious diseases. Leptospirosis might also be associated with renal diseases that are common in Sudan. We intended to explore the existence of human leptospirosis in suspected high risk patients in Khartoum, Sudan, via sero-screening random febrile patients and those undergoing renal dialysis.

\section{Methods}

This is a pilot exploratory study that was conducted in 6 months period from April to September of 2013. Hospitals were selected conveniently following a non-random sampling approach. A total of 119 febrile patients (with or without definitive diagnosis) and patients under renal dialysis were included and their serum specimens, clinical and demographic data were collected. Sera were screened qualitatively for the existence of anti-leptospiral IgM antibodies using rapid lateral flow immunosorbent assay. Ethical clearance and official permissions were obtained.

\section{Results}

Out of the total 119 patients, $57(47.9 \%)$ had end stage renal disease and were under dialysis at Renal Dialysis Unit in Asbab Charity Hospital in Bahri, 47 (39.5\%), were febrile with unknown origin attending the Tropical Medicine Hospital in Omdurman, and 15 (12.6\%) were febrile and were diagnosed as having malaria or typhoid and attended Yastabshiron Medical Centre and Bashauer Teaching Hospital. The overall prevalence of anti-leptospiral IgM antibodies among all 119 screened patients was $7 \%$. The prevalence among the 57 with end stage renal disease was $9 \%$, and among the 47 with fever of unknown origin was $6 \%$. The prevalence among the 15 with fever of known origin (diagnosed as malaria or typhoid) was $0 \%$. Almost all positive patients had recurring episodes of fever, are in close contact with livestock, were farmers and have natural untreated source for drinking water.

\section{Conclusion}

Leptospirosis is probably a common febrile condition and can be easily considered as one of the major causes of chronic kidney disease affecting people in this country. A national sero-screening for leptospirosis among those living in high risk geographical areas and those at occupational risk is highly recommended.

\section{Introduction}

Leptospirosis is the most widespread however underreported and neglected potentially fatal zoonotic bacterial infection affecting people worldwide $[1,2]$. It is estimated that more than 1.03 million cases and 58,900 deaths occur each year worldwide [3]. The highest burden of leptospirosis affects the tropics and 
sub-tropics especially following heavy rainfall and flooding [4]. Leptospirosis is caused by pathogenic species of the genus Leptospira that belongs to the phylum Spirochaetes [1].

For many developing countries in general, there are no data on the prevalence of human leptospirosis and for certain developing countries the data may be considered unreliable [5]. In Africa, little is known; the few existed reports indicated the presence of the disease in Morocco, Eritrea, and Ethiopia, but with no specific data. Kenya [6] reported an outbreak and Gabon [6] reported $15 \%$ seroprevalence. The very first report of the disease in Sudan was during the 70s in a variety of animals including camels, goats, sheep, dogs and mice [7]. With the exception of the preliminary data on the association of leptospirosis with endstage renal failure in Sudan [8], human leptospirosis has not been systematically studied in Sudan.

Leptospirosis can be an acute infection and can cause early complications like pulmonary haemorrhage and renal failure. It can also cause sub-acute and chronic complications in addition to long-term sequelae [9]. Infection results from direct or indirect exposure to urine from infected reservoir host animals that shed pathogenic leptospires which contaminate soils, surface waters, streams and rivers [2]. Humans are infected through mucous membranes, abrasions or cuts in the skin [10].

The laboratory diagnosis of leptospirosis can be established either by isolation of the intact live bacterium in specific culture media (which requires long incubation periods) or by detecting specific antibodies using serological techniques (the conventional ones are laborious and require freshly-made species-specific antigens) [11, 12], or by detecting Leptospira DNA using molecular techniques [13]. Since it was relatively easier and more accessible than culture and PCR, serology was mostly used historically for the diagnosis of leptospirosis [2].

Using archived samples collected from febrile patients in Thailand, the diagnostic capacity of two commercially available tests; an ELISA IgM and a rapid immune chromatographic test IgG were evaluated [14]. The sensitivities of IgM ELISA and ICT IgG were $61 \%$ and $83 \%$, respectivelyh'hh'yj. False positive ICT result occurred in one patient with influenza-B infection. The positive rates of both assays were high after the first week of onset of fever up to the third week of illness [14]. A large scale study involving 10 hospitals in North-Eastern Malaysia [15] identified a seroprevalence of $8 \%(n=84)$ of leptospirosis in 999 sera collected from febrile patient. They used IgM ELISA for screening and MAT for confirmation. Of the 1453 total patients that were clinically suspected for leptospirosis and were included in a 10 year retrospective sero-epidemiological survey of leptospirosis in a tertiary care hospital in New Delhi [16], 391 (27\%) were positive by IgM ELISA.

Since IgM antibodies are detectable in the blood from 5 to 7 days after the onset of symptoms [2], the qualitative sandwich immunoassay; Leptocheck ${ }^{\circledR}$ WB rapid lateral flow immunesorbent assay, was used in this study. It was designed for the sero-diagnosis of current or recent leptospirosis through detecting leptospira specific lgM in human serum, plasma or whole blood. This lateral flow immunesorbent assay was evaluated by Sehgal et al (2003) [17] at a primary health center in the Andaman Islands, where leptospirosis is endemic. All indices of validity and utility of lateral flow were similar to those of IgM ELISA and Lepto dipstick. 
In this limited-resource, self-financed pilot study, we intended to explore the existence of human leptospirosis in suspected high risk patients in Khartoum, Sudan, via sero-screening random febrile patients and those undergoing renal dialysis.

\section{Materials And Methods}

\section{Materials}

This is a pilot exploratory study. It was conducted in several hospitals in Khartoum, Sudan, during 6 months period from April to September of 2013. Hospitals were selected conveniently following a nonrandom sampling approach. A total of 119 patients were included; 57 of these suffered from end stage renal disease and were under dialysis at the Renal Dialysis Unit in Asbab Charity Hospital in Bahri area, 47 patients suffered from fever of unknown origin and were admitted to the Tropical Medicine Hospital in Omdurman area. The remaining 15 patients were febrile and suffering from malaria or typhoid and were attending Yastabshiron Medical Centre and Bashauer Teaching Hospital in Khartoum area.

Single blood samples were collected from the renal dialysis attendees and sera were separated. Ready serum samples were collected from the laboratories of remaining hospitals. Data were collected directly from patients undergoing renal dialysis; they were inquired about their age, sex, occupation, previous and current residence, contact with animals, source of drinking water and the occurrence of previous episodes of fever. Data were collected from hospital records for patients attending the remaining hospitals.

Screening for specific anti-leptospiral IgM antibodies in patient's sera was performed by the use of the rapid lateral flow immunosorbent assay Leptocheck ${ }^{\circledR}$ WB kit provided by Zephyr Biomedicals ${ }^{\odot}$. Sample storage and processing was performed at the serology laboratory of the Faculty of Medical Laboratory Sciences at the University of Medical Sciences and Technology (UMST). Informed consent was obtained from each patient at the renal dialysis unit prior to participation in the study. Permissions to use data anonymously and excess serum samples were obtained from hospitals authorities. The ethical clearance for conducting this study was obtained from the Ethical Committee Board of UMST in April of 2011.

\section{Leptocheck® WB rapid lateral flow immunosorbent assay}

This is a qualitative sandwich immunoassay designed for the sero-diagnosis of current or recent leptospirosis through detecting leptospira specific IgM in human serum, plasma or whole blood. The detection of leptospira infections caused by wide range of different serovars is maximized by the use of broadly reactive genus specific antigen. In the device, a membrane test is predispensed with anti-human IgM colloidal gold conjugate and leptospira genus specific antigens at test window $(T)$ and an anti-rabbit antiserum is predispensed at the control window (C).

The kit components were brought to room temperature before use. After labeling, $10 \mu \mathrm{L}$ of patient serum was transferred into sample port (A), 5 drops of running buffer was transferred to reagent port (B) and 
then the device was kept on bench at room temperature for 15 minutes. Test results were then read as follow;

A negative result is indicated when only one colored band appears in the control window (C). A positive result is indicated when, in addition to the band in control window (C), another red to purple band appears in the test window $(T)$. The later band indicates the presence of specific IgM antibodies to leptospira. The intensity of the test line depends upon the stage of the disease and the titer of the antibodies in patient serum.

Known positive and negative controls were applied prior to testing to ascertain the device quality. In order to maintain valid test conditions and proper result interpretations, all recommendations mentioned in the kit leaflet were followed.

\section{Results}

Out of the total 119 patients, 57 (47.9\%) suffered from end stage renal disease and were under dialysis at the Renal Dialysis Unit in Asbab Charity Hospital in Bahri area in Khartoum, Sudan. The average age of this group was $51 \pm 16$ years (range 17 to 80 years) and males represented $60 \%$ (34/57). Out of the 57 , anti-leptospiral IgM antibodies were detected in 5 (8.8\%). Their median age was 49 (range 42 to 67 years) and most $(4 / 5,80 \%)$ were males. All males $(100 \%)$ were farmers. With the exception of one male, all remaining 4 (80\%) patients had history of recurring episodes of fever and were living with domestic animals or have contacted them at some point. The sources of drinking water were as follow; the Nile for 3 out of the $5(60 \%)$, chlorinated water for one (20\%) and river canals for one $(20 \%)$. Three $(60 \%)$ out of the 5 patients were originally resident in Northern and Central states (Dongola/Rufaa) and currently in Bahri area in Khartoum, one (20\%) was resident in Juba and currently in Omdurman area in Khartoum, and one (20\%) was resident in Saudia Arabia and currently in Khartoum. One (20\%) of the positive patients had malaria at the time of sample collection; he was the one who was originally resident in Juba and uses river canals as source of drinking water.

The second group of patients, 47 out of total 119 (39.5\%), were febrile with unknown origin; they attended the Tropical Medicine Hospital in Omdurman area in Khartoum. Their average age was $40 \pm 16$ years (range 14 to 65 years) and male (46\%) to female (54\%) ratio was almost equivalent. Out of these 47 patients, anti-leptospiral IgM antibodies were detected in $3(6.4 \%)$. All 3 positive patients were above 50 years old and most $(2 / 3,67 \%)$ were males. All 3 had history of recurring episodes of fever and were in close contact with domestic animals. The males were farmers and were living in Algazira state in Central Sudan. The female was a housewife and was living in Dar Alsalam in Khartoum area.

The third group of patients, 15 out of total 119 (12.6\%), were febrile and were diagnosed as having malaria or typhoid. They attended Yastabshiron Medical Centre and Bashauer Teaching Hospital. Sera from these patients were not reactive (0\%) against anti-leptospiral IgM antibodies. 
The overall prevalence of anti-leptospiral lgM antibodies among all 119 screened patients was $7 \%$. The prevalence among the 57 with end stage renal disease was $9 \%$, and among the 47 with fever of unknown origin was $6 \%$. The prevalence among the 15 with fever of known origin (malaria/typhoid) was $0 \%$.

\section{Discussion}

Chronic kidney disease, which is a state of kidney damage or reduced kidney function lasting for three months or longer, is common in Sudan [18]. Several conditions can cause chronic kidney disease including diabetes, hypertension, or glomerulonephritis [19]. Leptospirosis is well known to be associated with kidney disease [20] but it has never been thoroughly investigated and is severely overlooked in most African countries, including Sudan. We believe this bacterium could be a major cause of chronic kidney disease in the country. In addition, symptoms of leptospirosis may mimic those of other endemic infections in Sudan such as malaria, typhoid, meningitis, hepatitis, or even dengue [20]. We, therefore, intended to explore its existence in possibly suspected high risk patients in Khartoum via sero-screening random febrile patients and those undergoing renal dialysis. Interestingly, anti-leptospiral IgM antibodies were detected in $7 \%$ out of all our 119 screened patients. Similarly, clinical researchers in Vanuatu (Pacific Islands) [21] wanted to investigate leptospirosis as a cause of non-malarial acute febrile illness and found a high seroprevalence of $6 \%$. Leptospira seroprevalence in a study in Western Uganda was 35\% [22]. They further reported a probable recent leptospirosis seroprevalence of $1.9 \%$ that was associated with having self-reported malaria within the past year [22]. Other studies reported varied leptospiral seroprevalence of $8.4 \%$ among febrile inpatients in North-Eastern Malaysia [23], 24\% among individuals at high-risk occupations in Morocco [24] and 38.5\% among miners in India [25]. Further, a study in Portugal revealed $46 \%$ of Sa o Miguel Island population has circulating anti-leptospiral antibodies [26].

A total of $9 \%$ out of all our 57 patients in renal dialysis and that of $6 \%$ out of the 47 patients with fever of unknown origin were seropositive for leptospiral specific $\lg \mathrm{M}$, indicating current or recent leptospirosis. Unfortunately, all patients undergoing dialysis were at end stage renal disease and those with fever of unknown origin were long term hospitalized. Knowing that the condition could have been easily reversed if the highly antimicrobial susceptible bacterial cause was identified early and treated-is heartbreaking. Therefore, sero-screening of suspected febrile patients or those with kidney disease should be a must in hospitals and renal centres in Sudan. Since this lateral flow immunochromatographic test kit can provide reliable results and is easy to perform, rapid, can be easily available and is cost effective, we recommend introducing it in the routine diagnostic laboratories, outpatient clinics and rural health centers in Sudan.

The majority of human cases of leptospirosis worldwide result from occupational exposure to water (or flood waters) or soil contaminated with animals urine [20,27]. People with relatively high incidence of infection -amongst others- are farmers, agricultural workers, animal handlers, and those exposed to flood waters $[20,27]$. Therefore, we wanted to explore these risk factors that could possibly be associated with leptospirosis among our population. A total of $80 \%$ out of the seropositive patients with end stage renal disease in our study had a history of recurring episodes of fever, and were living with domestic animals or have contacted them at some point. Most of these patients were farmers and a contaminated food and 
drink with animals and rodents urine can never be ruled out. These factors indicate and support the diagnosis of leptospirosis. In addition, the source of drinking water was the Nile River for $60 \%$ of these patients and $20 \%$ used river canals. The original residence for $60 \%$ was Dongola and Rofaa and $20 \%$ lived in Juba. Considering the fact that people living in these areas rely on the mentioned natural water sources without prior treatment suggest another possible risk for acquiring the infection. Higher risk activities in the study from Western Uganda included skinning cattle and living in close proximity to monkeys [22] whereas most of the seropositive high-risk individuals in the study from Morocco [24] were involved in poultry (37\%), market fish (26\%) and meat slaughterhouse workers (15\%).

Furthermore, the possibility that our sero-positive patients with fever of unknown origin might have consumed contaminated water by animal and rodents urine greatly arises since they were living in Aljazira and Dar Alsalam. The first area is known to be agricultural; facilitating a rich environment for the survival and spread of the disease, the second area has been urbanized by rural residents and the different water facilities have less quality control. All positive patients were middle aged or elderly. Many studies in the literature also reported a higher prevalence in this age group [20,27,28]. Male to female ratio was almost equivalent and this was also consistent with the findings of Rafizah et al(2012) [23] in their large scale study in North-Eastern Malaysia.

On the other hand, combined infections have been reported in literature [20, 27]; Chaudhry et al (2013) [29] reported hepatitis-E, malaria and dengue fever in $7 \%$ of patients with leptospirosis. In the present study, we identified co-infections in $25 \%$; one was co-infected with malaria and one with hepatitis-C. However, we did not identify anti-leptospiral antibodies $(0 \%)$ in the 15 patients with fever of known origin (i.e. malaria/typhoid).

Leptospirosis renal complications are present in our population indicated by a sero-positivity of approximately 2:1 among those with end stage renal disease (i.e. $62 \%$ ) relative to those with fever of unknown origin (i.e. 38\%). Yang et al (1997) [30] reported high prevalence of renal failure among patients with leptospirosis; they also reported fever and jaundice. Because of their finding, Yang and colleagues recommended that leptospirosis should be suspected in febrile patients with jaundice and renal failure, and we should do the same!

\section{Conclusion And Recommendation}

Leptospirosis is probably a common febrile condition and can be easily considered as one of the major causes of chronic kidney disease affecting people in this country. A national sero-screening for leptospirosis among those living in high risk geographical areas and those at occupational risk is highly recommended.

\section{Declarations}

\section{Ethics approval and consent to participate}


The ethical clearance for conducting this study was obtained from the Ethical Committee Board of the University of Medical Sciences and Technology in April of 2011. Permissions to collect specimens and data were granted from hospitals authorities. Patients who were contacted directly consented prior to participation. Data that were obtained from hospital files were kept anonymous at all stages of the study.

\section{Consent for publication}

All authors consented for the publication of this article.

\section{Availability of data and materials}

The datasets used and/or analyzed during the current study will be available from the corresponding author on reasonable request.

\section{Competing interests}

We, the authors, declare that we have no competing interests with respect to the research, authorship and/or publication of this article.

\section{Funding}

There is no financial support for this work.

\section{Authors' contributions}

WM imported kits from international market, approached patients and collected clinical specimens and data, performed laboratory investigations and followed-up all research reporting stages. NA developed research questions and design, performed statistical analysis and interpretation and wrote, edited and proofread the manuscript. All authors have read and approved the final manuscript.

\section{Acknowledgements}

We acknowledge all medical and laboratory workers at the Renal Dialysis Unit in Asbab Charity Hospital in Bahri area, the Tropical Medicine Hospital in Omdurman area, Yastabshiron Medical Centre and Bashauer Teaching Hospital in Khartoum area in Khartoum, Sudan, for facilitating specimen and data collection.

\section{References}

1. World Health Organization. Human leptospirosis: guidance for diagnosis, surveillance and control. Geneva: 2003.

2. Levett PN. Leptospirosis. Clin Microbiol Rev. 2001;14(2):296 \pm 326. https://doi.org/10.1128/CMR.14.2. 296-326.2001 PMID: 11292640 
3. Costa F, Hagan JE, Calcagno J, Kane M, Torgerson P, Martinez-Silveira MS, et al. Global Morbidity and Mortality of Leptospirosis: A Systematic Review. PLoS Negl Trop Dis. 2015; 9(9):e0003898. https://doi.org/10.1371/journal.pntd.0003898 PMID: 26379143

4. Pappas G, Papadimitriou P, Siozopoulou V, Christou L, Akritidis N. The globalization of leptospirosis: worldwide incidence trends. Int J Infect Dis. 2008; 12(4):351 \pm 7. https://doi.org/10.1016/j.jij.2007.09. 011 PMID: 18055245

5. Pappas G, Papadimitriou P, Siozopoulou V, Christou L, Akritidis N. The globalization of leptospirosis: worldwide incidence trends Int. J Infect Dis. 2008 Jul;12(4):351-7. E. pub 2007 Dec 4.

6. Feigin RD, Cherry JD, Demmler GJ, Kaplan SL, editors (2004). Leptospirosis Textbook of pediatric infectious diseases. 5th ed. Vol. 2. Philadelphia, Pennsylvania (USA): Saunders (Elsevier): 2004. p. $1708-22$

7. Shigidi MT. Animal leptospirosis in the Sudan. Br.Vet.J, 1974. 130(6): p. 528-531.

8. Mossaad E, EJI, Strutz F. The prevalence of leptospirosis in patients with end-stage renal failure in Sudan, in Bacterial, waterborne and emerging infectious diseases in North Africa and the Middle East. 2011, National Institute of Allergy and Infectious Diseases (NIAID): Nicosia, Cyprus.

9. Goris MG, Kikken V, Straetemans M, Alba S, Goeijenbier M, van Gorp EC, et al. Towards the burden of human leptospirosis: duration of acute illness and occurrence of post-leptospirosis symptoms of patients in the Netherlands. PLoS One. 2013; 8(10):e76549. https://doi.org/10.1371/journal.pone. 0076549 PMID: 24098528

10. Matono T, Kutsuna S, Koizumi N, Fujiya Y, Takeshita N, Hayakawa K, et al. Imported Flood-Related Leptospirosis From Palau: Awareness of Risk Factors Leads to Early Treatment. Journal of travel medicine. 2015; 22(6):422 \pm 4 . https://doi.org/10.1111/jtm.12241 PMID: 26503094

11. Hartskeerl RA, Collares-Pereira M, Ellis WA. Emergence, control and re-emerging leptospirosis: dynamics of infection in the changing world. Clin Microbiol Infect. 2011; 17(4):494 \pm 501. https://doi.org/10.1111/j.1469-0691.2011.03474.x PMID: 21414083

12. Schreier S, Doungchawee G, Chadsuthi S, Triampo D, Triampo W. Leptospirosis: current situation and trends of specific laboratory tests. Expert Rev Clin Immunol. 2013; 9(3):263 \pm 80 . https://doi.org/10.1586/eci.12.110 PMID: 23445200

13. Levett PN. Leptospirosis: a forgotten zoonosis? Clin Appl Immunol Rev. 2004; 4:435 \pm 48 .

14. Silpasakorn S, Waywa D, Hoontrakul S, Suttinont C, Losuwanaluk K, Suputtamongkol Y. Department of Medicine, Faculty of Medicine Siriraj Hospital, Mahidol University, Bangkok, Thailand. Performance of Leptospira immunoglobulin M ELISA and rapid immunoglobulin G immunochromatographic assays for the diagnosis of leptospirosis. J Med Assoc Thai. 2011 Feb;94 Suppl 1:S203-6.

15. Rafizah AA, Aziah BD, Azwany YN, Imran MK, Rusli AM, Nazri SM, Nikman AM, Nabilah I, Asma' HS, Zahiruddin WM, Zaliha I. A hospital-based study on seroprevalence of leptospirosis among febrile cases in northeastern Malaysia. Int J Infect Dis. pii: S1201-9712(13)00014-3. doi: 10.1016/j.jij.2012.12.012. 
16. Chaudhry R, Das A, Premlatha MM, Choudhary A, Chourasia BK, Chandel DS, Dey AB. Serological \& molecular approaches for diagnosis of leptospirosis in a tertiary care hospital in north India: A 10year study. Indian J Med Res. 2013 Apr;137(4):785-90.

17. Sehgal SC, Vijayachari P, Sugunan AP, Umapathi T. Source: National Leptospirosis Reference Centre, Regional Medical Research Centre, Indian Council of Medical Research, Post Bag no. 13, Port Blair 744101, Andaman \& Nicobar Islands, India. Field application of Lepto lateral flow for rapid diagnosis of leptospirosis. J Med Microbiol. Oct;52(Pt 10):897-901.

18. Mohamed Elhafiz Elsharif and Elham Garibballa Elsharif. Causes of end-stage renal disease in Sudan: a single-center experience. Saudi J Kidney Dis Transol 2011 Mar;22(2):373-6. PMID: 21422650

19. Teresa K. Chen, Daphne H. Knicely and Morgan E. Grams. Chronic kidney disease diagnosis and management; a review. JAMA. 2019 Oct 1; 322(13): 1294-1304. doi:10.1001/jama.2019.14745

20. Levett, P.N., Leptospirosis. Clin.Microbiol.Rev, 2001. 14(2): p. 296-326.

21. Pakoa JG, SoupeÂ-Gilbert M-E, Girault D, Takau D, Gaviga J, Gourinat A-C, et al. High incidence of leptospirosis in an observational study of hospital outpatients in Vanuatu highlights the need for improved awareness and diagnostic capacities. PLoS Negl Trop Dis 12(6): e0006564. https://doi.org/10.1371/journal.pntd.0006564

22. Dreyfus A, Dyal JW, Pearson R, Kankya C, Kajura C, Alinaitwe L, et al. Leptospira Seroprevalence and Risk Factors in Health Centre Patients in Hoima District, Western Uganda. PLoS Negl Trop Dis 10(8): e0004858. doi:10.1371/journal.pntd.0004858

23. Rafizah AA, Aziah BD, Azwany YN, Imran MK, Rusli AM, Nazri SM, Nikman AM, Nabilah I, Asma' HS, Zahiruddin WM, Zaliha I. A hospital-based study on seroprevalence of leptospirosis among febrile cases in northeastern Malaysia. Int J Infect Dis. pii: S1201-9712(13)00014-3. doi: 10.1016/j.ijid.2012.12.012.

24. Mohamed El Azhari, Mathieu Picardeau, Imad Cherkaoui, Mohamed Anouar Sadat, Houda Moumni, Kamal Marhoum El Filali, Hassan Ghazal, Abderrahmane Maaroufi, Salsabil Hamdi, Naima El Mdaghri, and Pascal Bourhy. Seroprevalence of Leptospirosis among High-Risk Individuals in Morocco. Interdisciplinary Perspectives on Infectious Diseases. Volume 2020, Article ID 5236045, 8 pages. https://doi.org/10.1155/2020/5236045

25. Sakkarai Mohamed Asha Parveen, Baskar Suganyaa, Muthu Sri Sathya, Alphonse Asirvatham Princy Margreat, Karikalacholan Sivasankari, Santhanam Shanmughapriya, Nicholas E. Hoffman, and Kalimuthusamy Natarajaseenivasan. Leptospirosis Seroprevalence among Blue Metal Mine Workers of Tamil Nadu, India. Am. J. Trop. Med. Hyg. 95(1), 2016, pp. 38-42 doi:10.4269/ajtmh.16-0095

26. Esteves LM, Bulho es SM, Branco CC, Mota FM, Paiva C, et al. Human Leptospirosis: Seroreactivity and Genetic Susceptibility in the Population of Sa o Miguel Island (Azores, Portugal). PLoS ONE 9(9): e108534. doi:10.1371/journal.pone.0108534

27. Guernier V, Goarant C, Benschop J, Lau CL. A systematic review of human and animal leptospirosis in the Pacific Islands reveals pathogen and reservoir diversity. PLoS Negl Trop. Dis 12(5): e0006503. 
https://doi.org/10.1371/journal.pntd.0006503

28. Feigin RD, Cherry JD, Demmler GJ, Kaplan SL, editors (2004). Leptospirosis Textbook of pediatric infectious diseases. 5th ed. Vol. 2. Philadelphia, Pennsylvania (USA): Saunders (Elsevier): 2004. p. $1708-22$

29. Chaudhry R, Das A, Premlatha MM, Choudhary A, Chourasia BK, Chandel DS, Dey AB. Serological \& molecular approaches for diagnosis of leptospirosis in a tertiary care hospital in north India: A 10year study. Indian J Med Res. 2013 Apr;137(4):785-90.

30. Yang CW, Pan MJ, Wu MS, Chen YM, Tsen YT, Lin CL, Wu CH, Huang CC. Leptospirosis: an ignored cause of acute renal failure in Taiwan. Am J Kidney Dis. 1997 Dec;30(6):840-5. 